\title{
\begin{tabular}{l|l} 
Mibraries & DSpace@MIT
\end{tabular}
}

\author{
MIT Open Access Articles
}

\section{Asymmetric multilevel outphasing transmitter using Class-E PAs with discrete pulse width modulation}

The MIT Faculty has made this article openly available. Please share how this access benefits you. Your story matters.

Citation: Chung, SungWon et al. “Asymmetric Multilevel Outphasing Transmitter Using classE PAs with Discrete Pulse Width Modulation." IEEE MTT-S International Microwave Symposium Digest (MTT), 2010. 264-267. (C) Copyright 2010 IEEE

As Published: http://dx.doi.org/10.1109/MWSYM.2010.5514707

Publisher: Institute of Electrical and Electronics Engineers (IEEE)

Persistent URL: http://hdl.handle.net/1721.1/73121

Version: Final published version: final published article, as it appeared in a journal, conference proceedings, or other formally published context

Terms of Use: Article is made available in accordance with the publisher's policy and may be subject to US copyright law. Please refer to the publisher's site for terms of use. 


\title{
Asymmetric Multilevel Outphasing Transmitter using Class-E PAs with Discrete Pulse Width Modulation
}

\author{
SungWon Chung, Philip A. Godoy, Taylor W. Barton, David J. Perreault, and Joel L. Dawson \\ Microsystems Technology Laboratories, Massachusetts Institute of Technology, Cambridge, MA 02139
}

\begin{abstract}
We present a high-efficiency transmitter architecture based on asymmetric multilevel outphasing (AMO), but with a new method of generating discrete amplitude levels from the constituent amplifiers. AMO and multilevel LINC (ML-LINC) transmitters improve their efficiency over LINC by switching the supplies of the power amplifiers (PAs) among a discrete set of voltages. This allows them to minimize the occurrence of large outphasing angles. However, it is also possible to generate a discrete set of amplitudes by varying the duty cycle of the waveform that drives the PAs. The chief advantage of this discrete pulse width modulation (DPWM) is hardware simplicity, as it eliminates the need for a fast, low-loss switching network and a selection of power supply voltages. We demonstrate this concept with a $48-\mathrm{MHz}, 20$ W peak output power AMO transmitter using a four-level DPWM. At peak output power, the measured power-added efficiency is $77.7 \%$. For a 16-QAM signal with a 6.5-dB peakto-average power ratio, the AMO prototype improves the average efficiency from $17.1 \%$ to $36.5 \%$ compared to the standard LINC system.
\end{abstract}

Index Terms- power amplifier (PA), outphasing, Class-E, pulse width modulation (PWM), asymmetric power combining, asymmetric multilevel outphasing (AMO), LINC, digital predistortion.

\section{INTRODUCTION}

Wideband RF power amplifiers (PAs) with high efficiency are of great importance in high-data-rate communications. Tremendous efforts to beat the linearity-efficiency tradeoff for PAs has led to a wide variety of architectures, including polar, outphasing, envelope tracking, feedforward, Cartesian feedback, predistortion, Doherty, RF pulse width modulation (PWM), and RF delta-sigma modulation [1].

Outphasing architectures [2], [3] are capable of transmitting very wideband signals and are thus suitable for wideband communication in multi-standard applications. However, the power efficiency of outphasing is poor at large power back-off, which is a critical drawback for wideband signals with high peak-to-average power ratios (PAPR). Outphasing with lossless power combining [4], [5] improves efficiency at the cost of reduced linearity and bandwidth.

This paper presents an outphasing transmitter architecture using Class-E PAs with discrete pulse width modulation (DPWM) for high-efficiency wideband RF

This work was sponsored by the Department of the Air Force under Contract FA8271-05-C-0002. Opinions, interpretations, conclusions, and recommendations are those of the author and are not necessarily endorsed by the United States Government. This work was also funded in part by the MIT Deshpande Center.

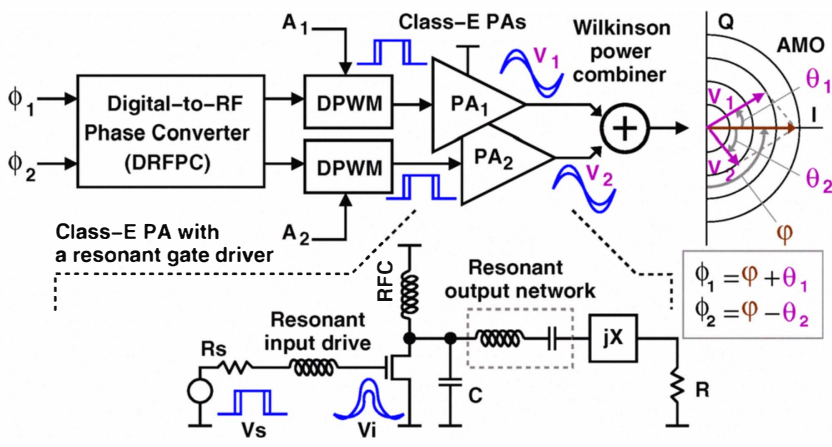

Fig. 1. Asymmetric multilevel outphasing (AMO) transmitter with discrete pulse width modulation (DPWM).

transmission. DPWM improves the classical outphasing power efficiency by allowing independent, discrete envelope amplitude changes in each of the two Class-E PAs. In contrast, conventional RF PWM Class-E PAs [6], [7] continuously modulate the input duty cycle over a large range with very fine resolution. One practical problem with the conventional PWM approach is that in order to achieve a wide dynamic range at $\mathrm{GHz}$ carrier frequencies, pulse widths on the order of a few picoseconds are required, which are difficult to generate. Furthermore, input pulses with very low duty ratios are not processed effectively by PAs so that the transmission of high PAPR results in poor linearity. With DPWM, these problems are overcome by restricting the duty cycles to a discrete set and avoiding small duty cycles. Outphasing is used for fine amplitude control and for generating very small amplitudes.

\section{Asymmetric Multilevel OutPhasing (AMO)}

The architecture of an AMO transmitter with DPWM is shown in Fig. 1. Each envelope amplitude of the two ClassE PAs is chosen from a discrete set of values such that the outphasing angle is minimized. The two PA outputs are combined based on the algorithm outlined in [8] in order to achieve high efficiency transmission over a wide range of output powers.

The use of DPWM with the AMO architecture is motivated by an efficiency analysis over the entire range of output power levels. The output power of the system can be modulated in two fundamental ways: either by varying the outphasing angles or the envelope amplitudes of the inputs to the Wilkinson combiner. In the case of outphasing, the amplitudes are held constant and the outphasing angle controls the output power as in a LINC architecture. 


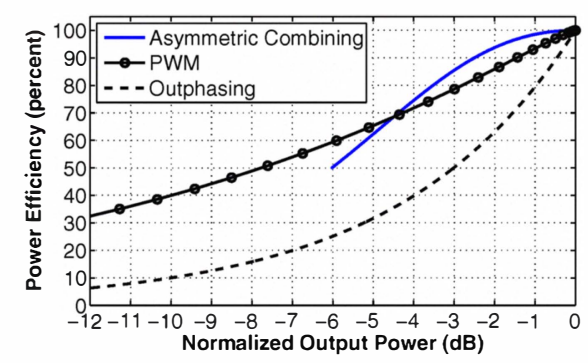

Fig. 2. Asymmetric power combining efficiency.

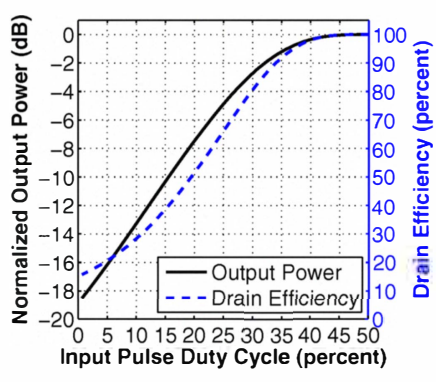

(a)

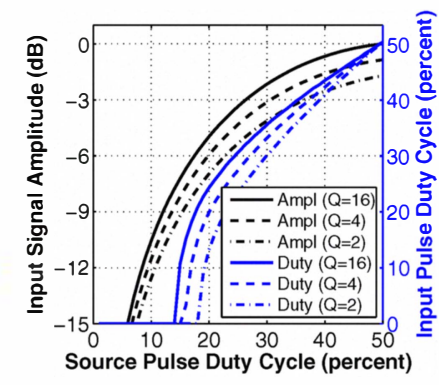

(b)
Fig. 3. (a) PA output power and efficiency with varying input duty cycles, (b) simulated impact of resonant PA drivers on the duty cycle and amplitude of PA input pulses, depending on input drive inductor Q.

Likewise, for envelope amplitude control the amplitudes are varied, either by modulating the PA supply voltage as in [8] or by PWM of the PA input as in this work. The efficiency of outphasing with an isolating combiner is compared to that of PWM of a single class-E PA in Fig. 2.

The efficiency of power combining with an ideal 2-way Wilkinson power combiner is given by

$$
\eta_{a}=\frac{\left(V_{1} \cos \theta_{1}+V_{2} \cos \theta_{2}\right)^{2}}{2\left(V_{1}^{2}+V_{2}^{2}\right)}
$$

where $V_{1}$ and $V_{2}$ are the amplitudes of the two RF sinusoid inputs whose outphasing angles are $\theta_{1}$ and $\theta_{2}$ (see Fig. 1 for notational convention). Because the AMO architecture allows $V_{1}$ and $V_{2}$ to be unequal, there is loss in the combiner for AMO even when there is no outphasing. This asymmetric combining effect is shown in Fig. 2 for the special case where the $\theta_{1}$ and $\theta_{2}$ are zero and the amplitude of $V_{2}$ is swept continuously between 0 and $V_{1}$.

AMO transmitters take advantage of the higher efficiency of envelope amplitude control by combining discrete amplitude changes with the fine envelope control provided by outphasing. In this paper, we present the use of PWM for amplitude control in an AMO transmitter. This approach can either replace or supplement the supply voltage modulation presented in [8].

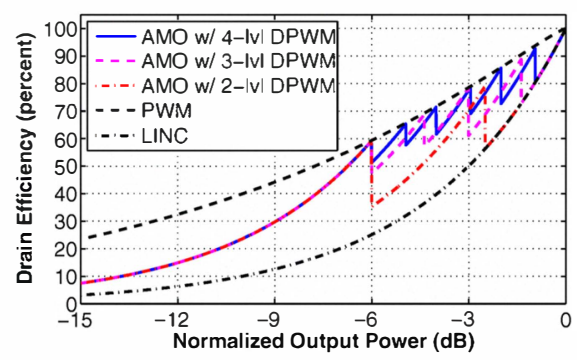

Fig. 4. Drain efficiency of ideal AMO-DPWM transmitters using a single supply.

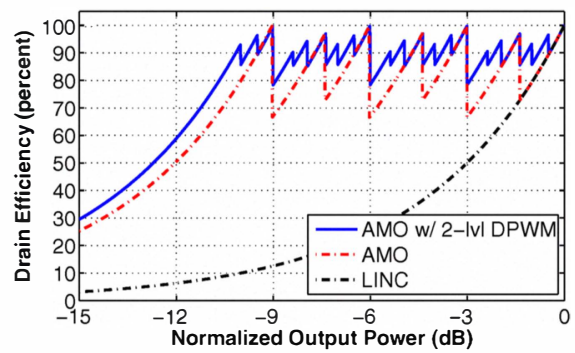

Fig. 5. Drain efficiency of ideal AMO-DPWM transmitters using a 4 level supply modulator (red line) vs. 4-level supply modulator combined with 2-level DPWM (blue line).

\section{Discrete Pulse Width Modulation (DPWM)}

DPWM provides discrete envelope amplitude modulation, without supply voltage modulation, to each of the two Class-E PAs used in outphasing. By limiting the input duty cycle variation to a finite number of discrete pulse widths, DPWM eliminates the need for extremely accurate pulse control.

DPWM can be implemented by (1) hard-switching inverter gate drive, (2) pulse input to resonant gate drive, (3) resonant gate drive with gate bias modulation. Each implementation has trade-offs. For example, the first two methods have limited power control range while gate bias modulation has a finite settling time. Likewise, a resonant input drive requires an inductor, making it best suited for a discrete implementation. Regardless of the DPWM implementation, the output power and drain efficiency of an ideal Class-E PA will have the relationship to duty cycle shown in Fig. 3(a).

\section{A. Impact of Resonant PA Driver}

The limited input duty cycle range of DPWM allows a resonant gate driver to be used. This is an important advantage over conventional PWM, as resonant gate drivers are often simpler to implement and more efficient than hard-switched drivers.

Fig. 3(b) shows the impact of a resonant PA driver on a $1-W$ Class-E PA with $5-\Omega$ gate resistance, $1-\mathrm{pF}$ gate capacitance, zero switch resistance, and ideal resonant 


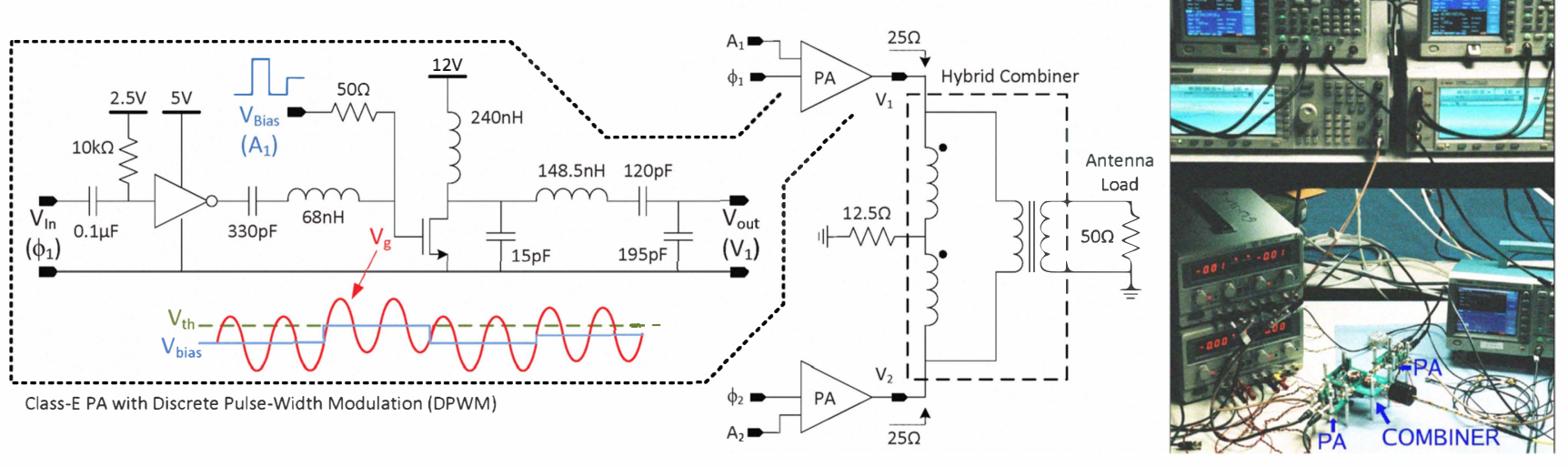

Fig. 6. Prototype 48-MHz AMO transmitter with DPWM.

output filter. The resonant PA input network with 50$\Omega$ source resistance and an inductor with a finite $\mathrm{Q}$, as shown in Fig. 1, shapes a source pulse $V_{s}$ into an input pulse $V_{i}$. When the source duty cycle gets smaller, the input amplitude gets smaller so that the resonant driver cannot turn on the main transistor of a Class-E PA. To provide sufficient PA input amplitude, the minimum input duty cycle should be larger than around $20 \%$. As a result, the maximum PA power back-off that can be achieved by DPWM is limited to less than $6 \mathrm{~dB}$. Additionally, because this input amplitude variation affects the Class-E PA output power, predistortion is necessary to provide a linear output.

\section{B. Efficiency of AMO with DPWM}

Fig. 4 shows the drain power efficiency of an ideal AMO-DPWM transmitter using a single supply. An ideal Class-E PA model [9] was used to get these results. When DPWM provides 6-dB power back-off, no more than four duty cycles $(23 \%, 27 \%, 32 \%, 50 \%)$ are necessary to get high efficiency. As shown in Fig. 5, this DPWM approach can also be combined with a multilevel supply modulator as in [8] to provide even higher overall efficiency with only two duty cycles $(35 \%, 50 \%)$.

\section{TRANSMitTER IMPLEMENTATION}

To demonstrate the feasibility of the AMO with DPWM system, a prototype was designed and implemented with discrete components at an operating frequency of $48 \mathrm{MHz}$. Fig. 6 shows a circuit schematic of the prototype, which is identical to that in [10] except for the gate driver. Each class-E PA was implemented using the ST Microelectronics PD57060 RF LDMOS, and was designed for a supply voltage of $12 \mathrm{~V}$ with $10 \mathrm{~W}$ output power. Thus the maximum output power of the prototype outphasing PA is 20W. Four parallel Fairchild NC7ZW04 CMOS inverters provide the gate drive. The power combiner used in the prototype consists of two 1:1 transformers, each implemented with 18AWG 5-turn bifilar windings

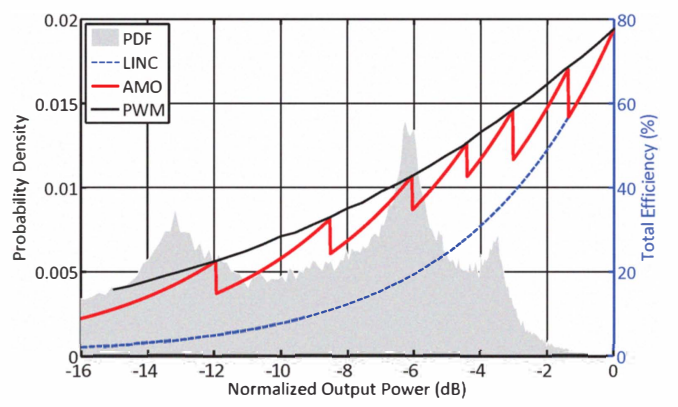

Fig. 7. Measured PWM power-added efficiency vs. output power, along with optimized DPWM levels for a 16-QAM signal with 6.5dB PAPR. The PDF of the 16-QAM signal is also shown.

on a Ferronics Cobalt-Nickel ferrite toroid core. The pulse width of each class-E PA is controlled by varying the gate bias voltage of the main transistor, as shown in Fig. 6.

\section{EXPERIMENTAL RESULTS}

Fig. 7 shows the measured PWM power-added efficiency (PAE) vs. output power for the prototype transmitter. For a given number of discrete pulse widths, this data can be used to find the optimum set of DPWM levels that maximize the efficiency for a given amplitude probability density function (PDF). In this work we chose to use four DPWM levels, and we tested our system with a 50-ksym/s 16-QAM signal with a PAPR of $6.5 \mathrm{~dB}$ and a carrier frequency of $48 \mathrm{MHz}$. The corresponding optimum efficiency curves for the AMO prototype are shown in Fig. 7. It can be seen that the AMO system with DPWM provides a significant efficiency improvement over the standard LINC system for a large output power range.

Fig. 8 shows the measured amplitude and phase of the output voltage at the fundamental of the RF output frequency versus the outphasing angle. The phase measurements are normalized to the output phase at the maximum output power. There are 10 different curves, each for a different combination of discrete pulse widths 

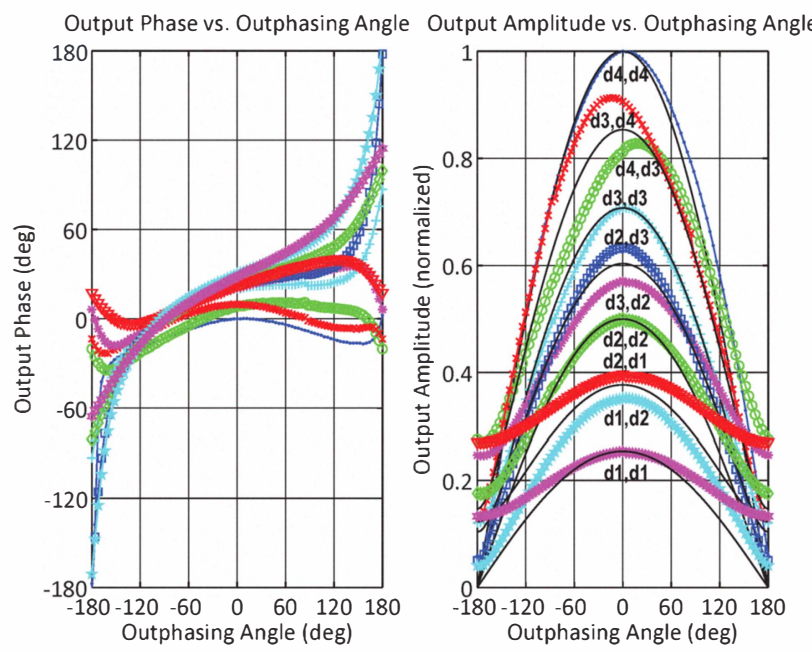

Fig. 8. Measured output amplitude and phase linearity for the prototype AMO system with DPWM. Each curve corresponds to a different combination of discrete pulse widths for the two outphased PAs. The ideal curves for the output amplitude are shown in black.
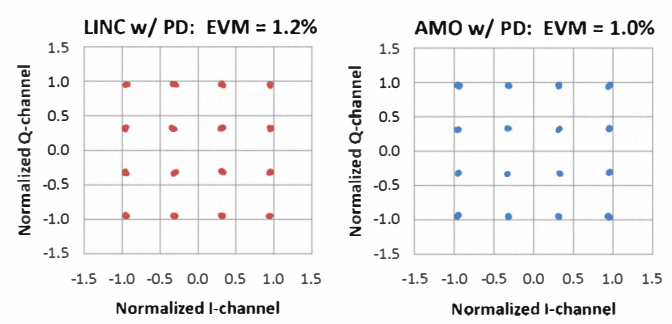

Fig. 9. Measured EVM of 50-ksym/s 16-QAM with 6.5-dB PAPR after predistortion.

for the 2 outphased PAs. Due to PA mismatch (which is the main source of the distortion), some combinations cannot achieve zero output amplitude even when both PAs use the same discrete pulse width and are completely out of phase, as can be seen in Fig. 8. Thus it is important that the discrete levels and combinations are chosen to achieve sufficent amplitude dynamic range while maximizing the overall efficiency. A lookup table constructed from the data in Fig. 8 is used to correct for the static nonlinearity.

To demonstrate the linearity of the system, we tested the prototype with a 50-ksym/s 16-QAM signal with a PAPR of $6.5 \mathrm{~dB}$ and a carrier frequency of $48 \mathrm{MHz}$. The digital baseband data generation and associated signal processing were performed in MATLAB and uploaded into the internal memory of arbitrary function generators. The baseband phase data for each PA was upconverted to $48 \mathrm{MHz}$ with an Agilent vector signal generator, and the system output was fed into an HP 89400 vector signal analyzer for spectrum and error vector magnitude (EVM) analysis. Fig. 9 shows the measured demodulated 16-QAM constellation of the prototype for both the standard LINC case and for the AMO-DPWM system after predistortion (PD). After

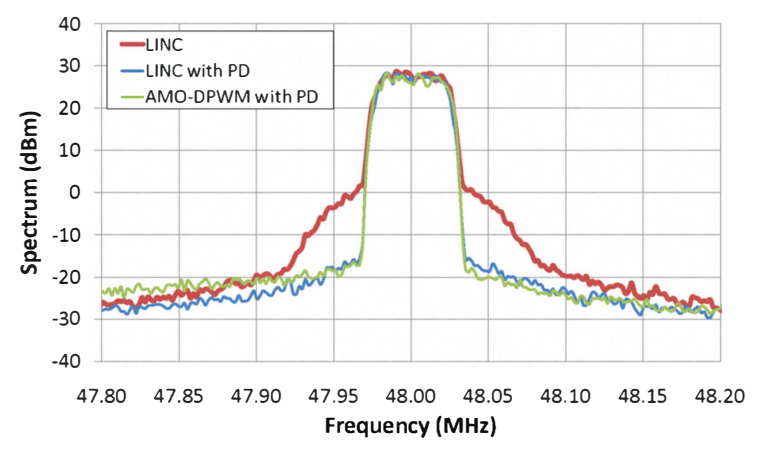

Fig. 10. Measured transmit spectrum of the 16-QAM signals.

predistortion, the EVM is reduced to $1.0 \%$. Fig. 10 shows the measured output spectrum for the 50-ksym/s 16-QAM transmission over a $48-\mathrm{MHz}$ carrier frequency. For the 16-QAM signal, the AMO system improves the overall efficiency from $17.1 \%$ to $36.5 \%$ compared to the standard LINC system, an efficiency improvement of more than $2 \mathrm{x}$.

\section{ConClusions}

The AMO transmitter using class-E PAs with DPWM was proposed to not only greatly increase transmitter efficiency but also enable wideband RF transmission. The DPWM technique was described as a method to provide an efficient coarse output envelope control, with outphasing providing the remaining fine envelope control. Finally, we demonstrate a 4-level AMO transmitter at $48 \mathrm{MHz}$ which improves the overall efficiency from $17.1 \%$ to $36.5 \%$ for a $50-\mathrm{ksym} / \mathrm{s} 16-\mathrm{QAM}$ signal with a PAPR of $6.5 \mathrm{~dB}$.

\section{REFERENCES}

[1] F. H. Raab, "Power amplifiers and transmitters for RF and microwave," IEEE Trans. Microwave Theory Tech., vol. 50, no. 3, pp. 814-826, Mar. 2002.

[2] H. Chireix, "High-power outphasing modulation," Proc. of the IRE, vol. 23, pp. 1370-1392, 1935.

[3] D. C. Cox, "Linear amplification with nonlinear components," IEEE Trans. Commun., pp. 1942-1945, Dec. 1974.

[4] S. Moloudi, K. Takinami, M. Youssef, M. Mikhemar, and A. Abidi, "An outphasing power amplifier for a software-defined radio transmitter," in ISSCC Dig. Tech. Papers, 2008, pp. 568-569.

[5] R. Beltran, F. H. Raab, and A. Velazquez, "HF outphasing transmitter using class-E power amplifiers," in Proc. IEEE Int'l Microwave Symp., 2009, pp. 757-760.

[6] J. Keyser, R. Uang, Y. Sugiyama, M. Iwamoto, I. Galton, and P. Asbeck, "Digital generation of RF pulsewidth modulated microwave signals using delta-sigma modulation," in Proc. IEEE Int'l Microwave Symp., 2002, pp. 397-400.

[7] J. S. Walling, S. S. Taylor, and D. J. Allstot, "A class-G supplymodulator and class-E PA in $130 \mathrm{~nm}$ CMOS," IEEE J. Solid-State Circuits, vol. 44, no. 9, pp. 2339-2347, Sept. 2009.

[8] S. Chung, P. A. Godoy, T. W. Barton, E. W. Huang, D. J. Perreault, and J. L. Dawson, "Asymmetric multilevel outphasing architecture for multistandard transmitters," in Proc. IEEE RFIC Symp., 2009, pp. 237-240.

[9] F. H. Raab, "Idealized operation of the Class E tuned power amplifier," IEEE Trans. Circuits Syst. II, vol. CAS-24, no. 12, pp. 725-735, Dec. 1977.

[10] P. Godoy, D. J. Perreault, and J. L. Dawson, "Outphasing energy recovery amplifier with resistance compression for improved efficiency," IEEE Trans. Microwave Theory Tech., Dec. 2009. 